\title{
The nitrogen vacancy in aluminium nitride
}

\author{
J M Vail ${ }^{1}$, D K Chevrier ${ }^{2}$, R Pandey ${ }^{3}$ and M A Blanco ${ }^{4}$ \\ ${ }^{1}$ Department of Physics and Astronomy, University of Manitoba, Winnipeg, MB, R3T 2N2, \\ Canada \\ 2 Department of Physics and Engineering Physics, University of Saskatchewan, Saskatoon, SK \\ S7N 5E2, Canada \\ ${ }^{3}$ Department of Physics, Michigan Technological University, Houghton, MI 49931, USA \\ ${ }^{4}$ Departamento de Química Física y Analítica, Universidad de Oviedo, E-33006-Oviedo, Spain
}

Received 25 October 2005, in final form 9 January 2006

Published 30 January 2006

Online at stacks.iop.org/JPhysCM/18/2125

\begin{abstract}
We have carried out a computational study for the nitrogen vacancy in charge states $+3,+2$ and +1 in $\mathrm{AlN}$ in the metastable zinc-blende phase. The vacancy and its four nearest-neighbour $\mathrm{Al}$ ions are treated as a molecular cluster, embedded in an infinite classical shell-model crystal. The following ground state properties, all of which are determinable from experiment, have been calculated: total spin, nearest-neighbour displacement, electron spin density at nearest-neighbour nuclei and breathing-mode force constant. The issue of disproportionation among the three charge states is also addressed. Most importantly, the optical excitation energies are evaluated.
\end{abstract}

\section{Introduction}

The group III nitrides AlN, GaN and InN are currently much studied because of their potential for technological device development [1]. Their optical properties are particularly of interest. As with all technological materials, useful properties depend heavily on the defect content of the material. Both the intrinsic materials and their defect properties have received extensive computational analysis, particularly by Van de Walle and co-workers, among others. Specifically with respect to point defects in AlN, we cite the extensive study by Stampfl and Van de Walle [2] and literature cited therein. The present work, dealing exclusively with the nitrogen vacancy in cubic zinc-blende structured AlN, presents new computational results on a number of experimentally relevant properties.

Most previous work on this system has used the density-functional analysis of electronic structure, applied in the supercell approach in which periodic boundary conditions are applied to a non-primitive unit cell of the crystal. The present work differs markedly in the modelling method applied. First, instead of density-functional based analysis, the unrestricted HartreeFock method is applied to a small molecular cluster, with second-order many-body perturbation 
theory correlation correction. Most importantly, this molecular cluster, containing the pointdefect, is embedded in an infinite crystal. Thus the present work deals with vanishingly low defect concentration, whereas the supercell approach deals with a concentration which is typically one atomic site in 32. This difference in defect concentration has the effect that the crystal field seen by electrons in and near the defect is markedly different in the two cases. In the supercell approach, the periodic array of point defects surrounding a particular one fails to represent accurately both the Madelung field of the ions that comprise the crystal, and the long-range polarization of the crystal that would be induced by charged point defects at low concentration. On the other hand, the embedded molecular cluster approach, while representing both Madelung and polarization features accurately, has its own shortcomings. Principal among these is the fact that the molecular cluster cannot be rigorously represented as a local region within a much larger crystal, in terms of many-electron effects. Furthermore, the limitation of the quantum cluster in the present work to nearest-neighbour ions obviously needs discussion and, ultimately, to be superseded by second-neighbour cluster calculations. These points will be addressed in section 2 .

In section 3 we give results, in three charge states of the nitrogen vacancy $V_{\mathrm{N}}$, for nearest-neighbour nuclear displacements from perfect crystal positions, electron spin densities at nearest-neighbour nuclei, breathing-mode force constants and optical excitation energies. The spins for $V_{\mathrm{N}}^{n+}$ (in the notation of Stampfl and Van de Walle) are 1, 0.5 and 0 (units $\hbar$ ) respectively for charge states $n=1,2$ and 3. Details of the electronic states involved in the optical transitions are very interesting, and are described. The disproportionation amongst the charge states, leading to instability of charge state 2, as described by Stampfl and Van de Walle, is quantified. Some further discussion of the results and method is given in section 4 . Conclusions are summarized in section 5.

\section{Model and computational method}

Aluminium nitride and related compounds can be thought of, from various viewpoints, as wide bandgap semiconductors, partly ionic semiconductors, or partly semiconducting ionic crystals. It is the latter viewpoint that guides the present work. We use an embedded molecular cluster approach, called the ICECAP method [3], in which the cluster, containing the point defect, is embedded in an infinite crystal. The embedding region is represented by the classical shell model for ionic crystals [4].

In the ICECAP method, the molecular cluster consists of bare nuclei and an appropriate set of electrons. Alternatively, core pseudopotentials may be used, but they are not used in the present work. With a reasonably flexible basis set for solution of the Fock equation for the electrons, there is a problem with the interface between the molecular cluster and the embedding shell-model region, namely that the minimum energy principle tends to push electronic density outward into the classical surrounding region in a way that does not occur in the real crystal. This can be realistically dealt with if the cluster is surrounded by ions that are represented entirely by pseudopotentials which have been derived for the perfect crystal. Best accuracy requires that this outer layer of pseudopotential ions lies at such a distance that the ions are not significantly affected by the defect. The time required for a Hartree-Fock calculation, and hundreds of them are required for the present study, increases sharply with the number of atoms, and the number of atoms increases sharply with cluster size. For the present work, we have taken the smallest cluster possible, namely the nitrogen vacancy site and its four nearest-neighbour aluminium ions. Our attempt [5] to dress this with second-neighbour nitrogen-ion pseudopotentials was unsuccessful, mainly because the pseudopotentials need to represent filled-shell atomic cores, in this case $\mathrm{N}^{3-}$ ions, which, as we shall see, is incompatible 
with the rest of the model. Similarly, our efforts [6] to incorporate the 12 second-neighbour nitrogen ions in the cluster were defeated by convergence problems in the self-consistent field treatment of the Fock equation. We do not doubt that this difficulty can eventually be overcome, but we think that in the meantime the current results provide a valuable baseline for the problem. A third approach used here, to represent the quantum mechanical effects of the embedding crystal, is to fix the orbitals of the outer layer of ions of the cluster so that they conform to the optimal orbital set in the perfect crystal. That this was not done in [7] accounts for the fact that the present results for $n=3$ do not agree with that earlier work.

The embedding shell-model crystal consists of point-charge ions interacting by Coulomb forces and by short-range shell-shell forces that combine Born-Mayer and van der Waals effects (a Buckingham potential). Each ion consists of two point charges, a core and a shell, harmonically coupled. The total ionic charge is taken to be known for each species, leaving the shell charges, along with shell-core force constants and Buckingham potential parameters, to be determined. The determination is made by fitting calculated bulk properties to experimental values. Ordinarily, for typical ionic crystals, the ionic charges are integers. For AlN and related materials, however, the crystal is not well represented by filled-shell integer values, $\mathrm{N}^{3-}$ and $\mathrm{Al}^{3+}$. For the shell model itself, fractional ionic charges are not a problem. We emphasize that, in the ICECAP method, the shell model is quite appropriate in the way it is used, in spite of its extreme simplicity. The reason is that, apart from quantum mechanical boundary effects for the molecular cluster, the embedding region only needs to represent the ionic charges and mechanical properties of the crystal, and in the case of charged point defects, as here, the dielectric polarization of an infinite region of crystal that is only weakly perturbed from the perfect-crystal state. The shell-model parameters, derived as they are from at most harmonic properties of the perfect crystal, allow the model to do this quite accurately.

The issue of effective ionic charge in AlN is addressed as follows. Starting from the results of a Hartree-Fock band structure calculation [8] we determine the net charge associated with each ionic species. This charge is determined by the method of Bader [9], in which the electronic density is integrated from the nucleus out to the surfaces of minimum electronic density that separate it from its bonded neighbours. For cubic AlN, the values turn out to be \pm 2.41 (units $e$, the proton charge). This approach to classical modelling for AlN, with the corresponding shell-model parameters, has been covered in a previous publication [7].

The fractional ionic charge creates a problem when it comes to specifying an embedded molecular cluster. The cluster is set up by starting with a shell-model version of the perfect crystal. One then removes shell-model ions that will be replaced by the defect molecular cluster, and replaces them by a set of nuclei and a set of electrons, keeping track of the energy involved, so as to be able to state an energy for the whole system: defect cluster plus embedding. To represent a defect $V_{\mathrm{N}}^{n+}$ in this way, the cluster must have a charge $n>0$, greater than the corresponding region of perfect crystal had before the cluster was created. The perfect-crystal cluster $\mathrm{Al}_{4} \mathrm{~N}$, with ionic charges \pm 2.41 , has a charge of 7.23. The cluster contains four $\mathrm{Al}$ nuclei with a total charge of 52. For the molecular cluster to have a charge of $(7.23+n)$, its electronic charge must be $(7.23+n-52)=(-44.77+n)$. For $n=1,2$ and 3 , the electronic charges must therefore be $-43.77,-42.77$ and -41.77 respectively. However, a molecular cluster calculation must be for an integral number of electrons, so rounding off the above numbers we see that the cluster should have 44, 43 and 42 electrons respectively, for $n=1,2$ and 3. Modelling calculations based on these numbers must, however, be recognized as under-estimating the net charge of the cluster region by 0.23 (units $e$ ).

The Al basis set from the band structure calculation which, along with the $\mathrm{N}$ basis set, was used in determining the ionic charge is also used here. As noted earlier [7], these atomic orbital basis sets describe an $\mathrm{Al}_{4} \mathrm{~N}$ cluster that is compatible with our embedding shell-model crystal 
Table 1. Computed ground-state properties of the nitrogen vacancy $V_{\mathrm{N}}^{n+}$ in charge states $n=1,2$ and 3 in zinc-blende AlN. Total spin $S$ (units $\hbar$ ); fractional nearest-neighbour Al ion displacements relative to perfect-crystal positions, $\left(d-d_{0}\right) / d_{0}$; electron spin density $\rho$ at relaxed nearest-neighbour $\mathrm{Al}$ ion nuclear positions (units $\hbar S a_{0}^{-3}$ ); effective force constant $K$ for breathing mode of vibration (units $\mathrm{eV} a_{0}^{-2}$ ); and lowest dipole allowed optical excitation energy $\Delta E$ (units eV): see also table 2.

\begin{tabular}{llllll}
\hline Defect & Spin $S$ & $\left(d-d_{0}\right) / d_{0}$ & $\rho$ & $K$ & $\Delta E$ \\
\hline$V_{\mathrm{N}}^{1+}$ & 1.0016 & 0.126 & $0.1377^{\mathrm{a}}$ & 51.1 & 1.69 \\
$V_{\mathrm{N}}^{2+}$ & 0.5031 & 0.030 & 0.1678 & 65.4 & 3.04 \\
$V_{\mathrm{N}}^{3+}$ & 0.0000 & 0.040 & 0 & 43.4 & 10.10 \\
\hline a Nuclei at $(0.563,0.563,0.563)$ and $(0.536,-0.563,-0.563) ;$ units, lattice spacing 2.1820 $\AA$. \\
b Nuclei at $(-0.563,-0.563,0.563)$ and $(-0.563,0.563,-0.563) ;$ units, lattice spacing 2.1820.
\end{tabular}

to good accuracy. Since the Madelung potential in the vacancy is highly attractive, the cluster basis set must contain vacancy-centred orbitals, and these must include at least $\mathrm{s}$ and $\mathrm{p}$ type because the vacancy site is not a centre of symmetry.

The process of replacing a perfect-crystal region in a shell model of the crystal by a molecular cluster is accomplished in two steps, in practice. In the first step, the perfectcrystal region is replaced by a shell-model representation of the defect. For this, the four nearest-neighbour $\mathrm{Al}$ ions start off as in the perfect crystal, with point charges +2.41 . Any electronic charge that shows up in the vacancy, and any change in the Al-ion charges that is associated with the defect, are represented by additional point-charge simulators. These pointcharge simulators must be such as to preserve the correct total charge of the cluster region. For example, in charge state +1 , where the defect region should have a total charge 8.23 , a vacancy simulator of -4.99 is compensated for by modifying the four Al charges from 2.41 to 3.305. These numbers are determined as follows. For the ground state of the defect, we want the polarization and deformation of the embedding crystal to be compatible with the HartreeFock representation of the cluster. This must be achieved by having the total energy of the crystal, cluster plus embedding, minimized. The polarization field which does this is set up by the vacancy simulator, whose charge is varied to optimization, while keeping the shell-model defect region correctly normalized as to total charge. Ultimately, two other parameters must be optimized along with the vacancy simulator, to self-consistently minimize the total energy. These are the range of the vacancy-centred orbitals and the nearest-neighbour distance. In the ICECAP method, the shell-model embedding relaxes to equilibrium with the cluster ions. These quantities are further discussed in section 3 .

\section{Computed results}

The computed results of most direct experimental interest are summarized in table 1. Each requires some discussion.

\subsection{Total spin $S$}

Defined by

$$
S(S+1)=\hbar^{-2} \cdot\left\langle\Psi\left|S_{o p}^{2}\right| \Psi\right\rangle
$$

where $\vec{S}_{\text {op }}$ is the total spin operator for the many-electron molecular cluster, and $\Psi$ is the manyelectron Hartree-Fock wavefunction. 


\subsection{Nearest-neighbour displacement}

The equilibrium fractional displacement $\left(d-d_{0}\right) / d_{0}$ of $\mathrm{Al}$ ions that are nearest neighbours of the vacancy, relative to the equilibrium $\mathrm{N}-\mathrm{Al}$ distance $d_{0}$ of the perfect AlN crystal in the zincblende structure. In the case $n=1$ for $V_{\mathrm{N}}^{n+}$, where the total spin is non-zero, the displacements are not all equal, but occur in two equal pairs, corresponding to the fact that the groundstate electronic charge distribution is of mixed parity: see Mulliken populations below, and table 3. However, in the present work, these two sets of coordinates have not been separately determined. Their differences are expected to be small because the Mulliken populations of the two sets of ions are so close: see table 3 . In the case $n=2$, the equality of the Mulliken populations for all four nearest neighbours in spite of non-zero spin comes from the fact that the spin $1 / 2$ is borne by a Fock orbital that is almost exactly p character, including the contributions from nearest-neighbour $\mathrm{Al}$ ions.

\subsection{Electron spin density $\rho(\vec{r})$ at nearest-neighbour nuclear positions}

In Hartree-Fock approximation, the electron spin density at $\vec{r}$ is $\hbar S$ times the electronic density. In units of $\hbar S$,

$$
\rho(\vec{r})=\left\{\sum_{j(\text { up })} \rho_{j}(\vec{r})-\sum_{j^{\prime}(\text { down })} \rho_{j^{\prime}}(\vec{r})\right\},
$$

where

$$
\rho_{j}(\vec{r})=\left|\psi_{j}(\vec{r})\right|^{2}
$$

In equation (3.2), $j$ (up) and $j^{\prime}$ (down) label occupied Fock eigenstates in the spin-up and spin-down manifolds respectively. In equation (3.3), $\psi_{j}$ is an occupied Fock eigenstate. The experimental isotropic hyperfine constant is determinable from $\rho$ evaluated at nuclear positions [10]. The two values given for the case $n=1$ correspond to the two inequivalent pairs of ions, as explained by the discussion in section 3.2 above.

\subsection{Breathing-mode force constant $K$}

The defected crystal has vibrational modes that are radially damped with distance from the point defect. The effective force constant for such a localized breathing mode can be estimated from:

$$
E_{\text {tot }}(D)=\frac{1}{2} K\left(D-D_{0}\right)^{2}
$$

where $E_{\text {tot }}$ is the total energy of the defected crystal, relative to equilibrium, $D$ is the distance of nearest-neighbour ions from the vacancy site, and $D_{0}$ is the equilibrium nearest-neighbour distance in the defect. Here, the small difference in the Mulliken populations of two pairs of neighbours in spin state $n=1$ and its attendant effect on local mode dynamics is neglected, as for nearest-neighbour equilibrium positions: see section 3.2 above. A rough estimate of the breathing-mode frequency can be obtained from

$$
\omega=(K / m)^{1 / 2}
$$

where in this case $m$ might be taken to be four times the mass of a nearest-neighbour $\mathrm{Al}$ ion. 
Table 2. Computed optical excitation energies $\Delta E$ for $V_{\mathrm{N}}^{n+}$ in z.b. AlN: Hartree-Fock, approximate correlation correction and total (units $\mathrm{eV}$ ).

\begin{tabular}{lccc}
\hline Defect & $\Delta E$ (Hartree-Fock) & Correlation & $\Delta E$ (total) \\
\hline$V_{\mathrm{N}}^{1+}$ & 2.03 & -0.34 & 1.69 \\
$V_{\mathrm{N}}^{2+}$ & 3.20 & -0.16 & 3.04 \\
$V_{\mathrm{N}}^{3+}$ & $11.01^{\mathrm{a}}$ & -0.91 & $10.10^{\mathrm{a}}$ \\
\hline a Corrected for significant spin mixing.
\end{tabular}

Table 3. Mulliken populations of $V_{\mathrm{N}}^{n+}$ in z.b. AlN in ground and optically excited states, and at nearest-neighbour Al sites.

\begin{tabular}{|c|c|c|c|c|c|c|}
\hline & \multicolumn{2}{|c|}{ Spin up } & \multicolumn{2}{|c|}{ Spin down } & \multicolumn{2}{|c|}{ Total } \\
\hline & Ground & Excited & Ground & Excited & Ground & Excited \\
\hline $\mathrm{Al}(1,1,1)$ & 4.8566 & 4.8693 & 5.0139 & 5.0007 & 9.8705 & 9.8699 \\
\hline $\mathrm{Al}(-1,-1,1)$ & 4.8090 & 4.7701 & 4.9882 & 5.0080 & 9.7972 & 9.7782 \\
\hline $\mathrm{Al}(-1,1,-1)$ & 4.8090 & 4.7701 & 4.9882 & 5.0080 & 9.7972 & 9.7782 \\
\hline $\mathrm{Al}(1,-1,-1)$ & 4.8566 & 4.8692 & 5.0139 & 5.0007 & 9.8705 & 9.8699 \\
\hline$V_{\mathrm{N}}^{1+}(0,0,0)$ & 3.6688 & 3.7212 & 0.9959 & 0.9826 & 4.6646 & 4.7038 \\
\hline $\mathrm{Al}$ & 4.9829 & 4.9652 & 5.0073 & 5.0277 & 9.9902 & 9.9929 \\
\hline $\mathrm{Al}$ & 4.9829 & 4.9551 & 5.0073 & 5.0057 & 9.9902 & 9.9208 \\
\hline $\mathrm{Al}$ & 4.9829 & 4.9551 & 5.0073 & 5.0057 & 9.9902 & 9.9208 \\
\hline $\mathrm{Al}$ & 4.9829 & 5.0204 & 5.0073 & 4.9854 & 9.9902 & 10.0094 \\
\hline$V_{\mathrm{N}}^{2+}$ & 2.0684 & 2.1807 & 0.9707 & 0.9754 & 3.0391 & 3.1560 \\
\hline $\mathrm{Al}$ & 5.0240 & 4.9958 & 5.0240 & 4.9769 & 10.0480 & 9.9724 \\
\hline $\mathrm{Al}$ & 5.0240 & 4.9479 & 5.0240 & 5.0090 & 10.0480 & 9.9569 \\
\hline $\mathrm{Al}$ & 5.0240 & 4.9950 & 5.0240 & 4.9997 & 10.0480 & 9.9947 \\
\hline $\mathrm{Al}$ & 5.0240 & 4.9796 & 5.0240 & 4.9948 & 10.0480 & 9.9745 \\
\hline$V_{\mathrm{N}}^{3+}$ & 0.9041 & 1.0816 & 0.9041 & 1.0199 & 1.8081 & 2.1015 \\
\hline
\end{tabular}

\subsection{Optical excitation energy $\Delta E$}

The optical excitation energy given is the lowest dipole allowed excitation from the manyelectron ground state to a many-electron excited state. The values given in table 1 include correlation correction determined by second-order Rayleigh-Schrödinger many-body perturbation theory, approximated using the virtual Fock manifold. In table 2, Hartree-Fock and correlation corrections are given separately. For $n=3$ the excited state as calculated contains significant mixing between the pure spin-zero state and the forbidden spin-one state lying $3.46 \mathrm{eV}$ below it. The forbidden state must therefore be projected out, in calculating the excited state energy. This has been done, before and after correlation correction. The excited states are calculated on the basis of the Franck-Condon approximation, in the following sense. It is assumed that the optical transition occurs faster than the relaxation of the surrounding crystal. Thus, in the present work, not only are nearest-neighbour nuclei and all shell-model cores frozen in the positions determined by ground-state equilibrium, but so are shell positions. An attempt to re-optimize the excited-state vacancy-centred atomic orbitals was abandoned, as it led to orbitals that strongly overlapped the ions, without being either well localized in the vacancy or broadly diffuse in the embedding region, suggesting large basis set superposition error in the excited states. For the same reason, it has been necessary to keep the exponential coefficients of optimized vacancy-centred s- and p-type orbitals the same in both ground and excited states. A redistribution of electronic density between vacancy and nearest-neighbour 
$\mathrm{Al}$ ions, and among the $\mathrm{Al}$ ions, does occur in the transition, however.

\subsection{Disproportionation}

Disproportionation refers to the relative stability of different charge states within the crystal. In the present case, the nitrogen vacancy, it can be quantified by the energy difference between two vacancies of charge +2 each, and two vacancies of charges +1 and +3 , respectively. Schematically,

$$
2 V_{\mathrm{N}}^{2+} \rightarrow\left(V_{\mathrm{N}}^{1+}+V_{\mathrm{N}}^{3+}+E_{\mathrm{D}}\right)
$$

where $E_{\mathrm{D}}$ is the disproportionation energy (intrinsically positive). Negative $E_{\mathrm{D}}$ would indicate that $n=2$ was the more stable species. In the present case, $E_{\mathrm{D}}$ can be evaluated from the total energies of the three charge states, all calculated in exactly the same model. The result is

$$
E_{\mathrm{D}}=1.39 \mathrm{eV} \text {. }
$$

\section{Discussion of results}

\subsection{Fractional nearest-neighbour displacement}

Stampfl and Van de Walle obtained values of $2.3 \%, 9.1 \%$ and $17.3 \%$ for the cases $n=1,2$ and 3 respectively. Surely, in our work as in theirs, the positive sign in all three vacancy charge states is reasonable: the net positive charge causes mutual Coulomb repulsion among the $\mathrm{Al}$ ions. The monotonic trend to larger values with increasing net charge is also easily understood on that basis. In our work the results are quite different: $12.6 \%, 3.0 \%$ and $4.0 \%$. We are not able to offer a simple explanation of this difference. Apart from the considerable difference in modelling approach, we note that as we change the defect charge from 1 to 2 to 3 , we find that the charge of each of the four nearest-neighbour $\mathrm{Al}$ ions remains at +3 , within a variation of less than $2 \%$. The charge increments take place almost entirely in the well-localized vacancy region. If another approach were to have the charge increments largely associated with the nearest-neighbour Al ions, then one might well obtain quite different results because of the complicated relationship between atomic arrangement and electronic distribution. See also section 4.3 .

\subsection{The optical transition}

The Fock eigenstates at the top of the occupied manifold in both the ground and excited states are dominated by vacancy-centred orbitals, a fact that is reflected in table 3 . In all three charge states, the vacancy s-type orbital is doubly occupied by a spin-up-spin-down pair of electrons. In charge state $n=2$ there is, in addition, a spin-up p-type electron, at a considerably higher energy. In charge state $n=1$, there are two nearly degenerate p-type spin-up electrons as well as the s-type spin pair. The fact that all electrons beyond those that maintain the filled-shell ionic charge of +3 for the nearest-neighbour Al ions are localized in the vacancy makes the defect appear and behave much like an F-type centre, in all three charge states. The 'excess electrons' are now those that are surplus to charge +3 for the $\mathrm{Al}$ ions, rather than as in the $\mathrm{F}$ centre, where they are surplus to the crystal with a simple anion vacancy. The fact of increasing tendency to definite p- or s-type character at the top of the valence band with increasing charge reflects increasing localization arising as it does from increasing strength of the Coulomb potential. The localization can be quantified by defining the range $R$ of a Gaussian function (of which our atomic orbitals are formed) to be

$$
R=(2 \alpha)^{-1 / 2}
$$


Table 4. Mulliken populations for $V_{\mathrm{N}}^{n+}$ in z.b. AlN for vacancy-centred $\mathrm{s}, \mathrm{p}_{x}, \mathrm{p}_{y}$ and $\mathrm{p}_{z}$ orbitals in both ground and excited states.

\begin{tabular}{|c|c|c|c|c|c|c|}
\hline & \multicolumn{2}{|c|}{ Spin up } & \multicolumn{2}{|c|}{ Spin down } & \multicolumn{2}{|c|}{ Total } \\
\hline & Ground & Excited & Ground & Excited & Ground & Excited \\
\hline \multicolumn{7}{|c|}{$n=1$} \\
\hline$s$ & 1.0935 & 1.0982 & 0.9860 & 0.9803 & 2.0804 & 2.0786 \\
\hline$x$ & 1.2843 & 1.2831 & 0.0083 & 0.0016 & 1.2927 & 1.2847 \\
\hline$y$ & 0.5850 & 0.6501 & 0.0003 & 0.0003 & 0.5853 & 0.6505 \\
\hline$z$ & 0.7060 & 0.6998 & 0.0003 & 0.0003 & 0.7063 & 0.6901 \\
\hline \multicolumn{7}{|c|}{$n=2$} \\
\hline$s$ & 0.9338 & 0.9435 & 0.9686 & 0.9702 & 1.9024 & 1.9137 \\
\hline$x$ & 1.1332 & 0.0012 & 0.0007 & 0.0008 & 1.1338 & 0.0020 \\
\hline$y$ & 0.0007 & 0.6180 & 0.0007 & 0.0022 & 0.0014 & 0.6202 \\
\hline$z$ & 0.0007 & 0.6180 & 0.0007 & 0.0022 & 0.0014 & 0.6202 \\
\hline \multicolumn{7}{|c|}{$n=3$} \\
\hline$s$ & 0.9023 & 0.0003 & 0.9023 & 1.0177 & 1.8046 & 1.0180 \\
\hline$x$ & 0.0006 & 0.5682 & 0.0006 & 0.0008 & 0.0012 & 0.5691 \\
\hline$y$ & 0.0006 & 0.1214 & 0.0006 & 0.0007 & 0.0012 & 0.1221 \\
\hline$z$ & 0.0006 & 0.3917 & 0.0006 & 0.0006 & 0.0012 & 0.3923 \\
\hline
\end{tabular}

where $\alpha$ is the Gaussian exponential coefficient, and the factor 2 comes from considering the square of the atomic orbital, corresponding to an electronic density. The ranges of vacancycentred orbitals, determined variationally, the same for s- as for p-type, are $0.88,0.66$ and 0.60 (units: perfect-crystal nearest-neighbour spacing) for $n=1,2$ and 3 respectively.

The selection rules for allowed electric dipole transitions in the nitrogen vacancy are affected by the absence of a centre of inversion for the molecular cluster and by the crystalline embedding. In each case, we have calculated the transition energy by de-occupying the highest occupied ground state Fock eigenstate and occupying the lowest virtual eigenstate. The details of the transition can be assessed from three sources: the Mulliken populations for Al ions in ground and excited states of the defect, corresponding data for vacancy-centred orbitals and details of the highest occupied Fock eigenstates in ground and excited states. The first two are collected in tables 3 and 4. In thinking about these data, two things must be borne in mind. First, Mulliken populations are not always a simple indication of the number of electrons in an orbital or on a site because of orbital overlap. Second, the Fock eigenstates, representing single-electron orbitals, are in fact molecular orbitals, typically with contributions from several, if not all, atomic orbitals in the calculations. With these points in mind, we have the following comments.

For charge state $n=1$, ground and excited states as calculated are almost pure spin-one states (spins 1.0016 and 1.0011 respectively: units $\hbar$ ). From table 4 we see that in the ground state the spin-paired vacancy-centred s-type electrons are fairly convincing, as is a spin-up $\mathrm{p}_{x}$ type electron, and somewhat more than one $\mathrm{p}_{y}-\mathrm{p}_{z}$ hybrid electron. There is almost no change in the vacancy-centred orbital occupancies upon excitation, and yet the calculated excitation energy is $2.03 \mathrm{eV}$ (see table 2). We must turn to the Fock eigenstates for the top of the occupied manifold in ground and excited states, not shown here, for an explanation. It turns out that the three highest spin-up eigenstates are $\mathrm{s}^{-}, \mathrm{p}_{y}-\mathrm{p}_{z}$ - and $\mathrm{p}_{x}$-type vacancy-centred molecular orbitals, each with significant s-type admixtures from the nearest-neighbour Al ions. In the ground state, the order of these molecular orbitals is as given, whereas in the excited state they are in the order $\mathrm{s}, \mathrm{p}_{x}$ and $\mathrm{p}_{y}-\mathrm{p}_{z}$. Furthermore, in the transition, the $\mathrm{p}_{x}$-type orbital has its sign 
changed relative to the ground state. The $\mathrm{s}-$ and $\mathrm{p}_{y}-\mathrm{p}_{z}$-type orbitals are qualitatively unchanged and both are unsymmetrical as to their $\mathrm{Al}$ orbital content, the $\mathrm{p}_{y}-\mathrm{p}_{z}$ orbital strongly so. The result is an excitation in which an orbital that has odd parity with respect to inversion along the $x$-axis reverses direction in the presence of $\mathrm{s}$ - and $\mathrm{p}_{y}-\mathrm{p}_{z}$-type orbitals that are qualitatively unchanged in the transition and that are unsymmetrical with respect to this inversion due to their $\mathrm{Al}$ content.

For charge state $n=2$, the picture from table 4 is much simpler than for $n=1$. Here again, the ground and excited state spins are almost pure, in this case spin- $1 / 2$. Specifically the calculated values are 0.5031 and 0.5033 respectively (units: $\hbar$ ). From table 4 we again see the spin-paired vacancy-centred s-type electrons, plus a $p_{x}$-type electron in the ground state and a $\mathrm{p}_{y}-\mathrm{p}_{z}$-type electron in the excited state. Although the ground-state $\mathrm{p}_{x}$-type electron is almost pure $\mathrm{p}$ type, the excited-state $\mathrm{p}_{y}-\mathrm{p}_{z}$-type electron has strongly mixed parity because of substantial contributions from a pair of nearest-neighbour $\mathrm{Al}$ ions.

For charge state $n=3$, we only have Mulliken population and Fock eigenstate data for the mixed-spin excited state, from which we have projected the forbidden spin-flip excited state to obtain the dipole-allowed excited state energy. For the ground state, the highest occupied Fock eigenstate is almost pure even-parity vacancy s-like. The highest occupied mixed-spin excited-state Fock eigenvector is a combination of $\mathrm{p}_{x^{-}}, \mathrm{p}_{y^{-}}$and $\mathrm{p}_{z}$-type vacancy orbitals, with relatively weak parity-breaking $\mathrm{Al}$ atomic orbital components. Thus charge state +3 is quite like the common s- to p-type transition seen for hydrogenic atoms, even as viewed through the mixed-spin excited state solution. We believe that this reflects the relatively strong localization, compared to charge states +2 and +1 , due to the extra strength of the potential well at the vacancy site. That is, the electrons bound in the vacancy are relatively unaffected by the ions of the surrounding crystal, except for its Madelung field. The calculated excitation energy for $n=3$ is $10.1 \mathrm{eV}$. In the next section we discuss some implications of such a large computed value.

\subsection{Comments on the model}

Some features of the model we have used require comment.

First, the use of classical modelling lacks the overlap and Pauli repulsion effects among electrons arising from the embedding region. This raises the question of the accuracy of a highly localized molecular cluster to simulate the defect. In the present work, limited to nearestneighbour atoms, we compensate for the lack of quantum mechanical embedding by limiting the $\mathrm{Al}$ atomic-orbital basis set to that which has been derived for a perfect AlN crystal. This limits the variational flexibility in such a way as to exclude spurious excursions of the electrons into the classical shell-model region. As we have mentioned, application of this approach to an embedded perfect-crystal molecular cluster shows good compatibility with the embedding region in terms of equilibrium spacing. Such an approach in the past has also led to widespread agreement with experimental results for point defects in strongly ionic crystals, and is therefore reasonable for an initial theoretical analysis of this system.

We have mentioned that this defect, with electrons in the vacancy, has similarities with $\mathrm{F}$ centres. It is well known that, for F centres, the possibility of highly diffuse localized states overlapping a large number of ions needs to be considered (see for example [11] and other references therein). In the form of modelling used here, an indication of such diffuseness is the existence of an energy minimum at several atomic distances from the vacancy, as a function of range (equation (4.1)) of vacancy-centred orbitals. For both ground and excited state orbitals, we found a monotonic increase in energy as a function of range in all three charge states, suggesting that both states are well localized within the vacancy. We say 'suggesting' because 
the issue is not fully resolved until polaronic corrections have been applied (as for example in [11]).

The $\mathrm{Al}$ basis set that has been used is of the form (8511/511/1). One might be concerned about the accuracy of the excited-state results in this case, particularly if the single-Gaussian $3 \mathrm{~s}, 3 \mathrm{p}$ and $3 \mathrm{~d}$ polarization orbitals fail to provide an accurate picture of the function-space region available for the transition. This is particularly the case for charge state +3 , where our calculated excitation energy seems to be quite high. We have therefore introduced threecomponent contractions for $3 \mathrm{~s}, 3 \mathrm{p}$ and $3 \mathrm{~d}$ orbitals [12], the first two directly from Huzinaga's compilation [13] for the free neutral $\mathrm{Al}$ atom. The $3 \mathrm{~d}$ orbital is concocted by scaling from $\mathrm{Kr}$. For charge state +3 , all qualitative features of the results are the same as, and numerically close to, those with single-component $3 \mathrm{~s}, 3 \mathrm{p}$ and $3 \mathrm{~d}$ orbitals, presented in section 3 . For charge states +2 and +1 , the excitation energies are qualitatively unchanged and dielectric polarizations are similar to the results reported here. The transition still predominantly involves vacancycentred orbitals. The question of diffuseness of vacancy-centred orbitals remains outstanding with this basis set. Mulliken populations in the vacancy are somewhat affected, but fractional nearest-neighbour displacements are significantly changed, to $7.0 \%$ and $9.6 \%$ respectively. We conclude that use of more accurate $\mathrm{Al} 3 \mathrm{~s}, 3 \mathrm{p}$ and $3 \mathrm{~d}$ orbitals does not materially improve on the results reported in section 3 .

It is an admitted weakness of the present work that second-neighbour $\mathrm{N}$ electrons are not included in the Hartree-Fock cluster. At the same time, there are three details of the present results that might be questioned. These are the high excitation energy for $n=3$, the relatively large nearest-neighbour relaxation for $n=1$ and the fact that four electrons are bound within the vacancy for $n=1$, two of them not spin paired. It is interesting to consider the possible role that nitrogen orbitals would play in determining these quantities.

In a deep potential well, which we have for charge-state 3 , it is understandable that the excitation energy will be high. However, instead of being vacancy-like, the excited state might be thought to be significantly influenced by $\mathrm{N}$-orbital content, or be dominated by $\mathrm{N}$ orbitals. While the chemical character of valence and conduction band edges, as well as the bandgap, can be radically affected by a defect $[14,15]$, it is worthwhile to examine the perfect crystal case. We have re-examined the earlier study of the band structure of zinc-blende AIN [8], and find the bottom of the conduction band to be predominantly of Al-orbital character, but with about $40 \% \mathrm{~N}$ content. Indeed, a calculation of a perfect-crystal $\mathrm{Al}_{4} \mathrm{~N}$ embedded cluster reveals the lowest virtual state to be similar: dominated by Al p-type orbitals, but with non-trivial $\mathrm{N}$ p-type content. Thus there is reason to suspect that this excitation may involve the secondneighbour nitrogens. We have already shown that throughout our calculations the nearestneighbour $\mathrm{Al}$ ions hold their charge (in terms of Mulliken populations) at +3 . They resist taking on electronic charge in the $n=3$ excitation, possibly reflecting the robustness of the filled-shell configuration. By the same reasoning, one might expect second-neighbour nitrogens to be receptive, moving their charges toward -3 from perfect-crystal values of -2.41 . And again, it is likely that even in the presence of nitrogen electrons, the two electrons drawn into the vacancy come from the nearest-neighbour aluminium ions, as in the present calculation, rather than from nitrogen ions. We recall that our calculated excitation energy is derived from many-body, not single-particle, energies. The same applies to the experimental band width, which is likely $\sim 6-7 \mathrm{eV}$, since it is known to be $6.28 \mathrm{eV}$ in wurtzite AlN (see [2]). The single-particle value from the Hartree-Fock band structure approach [8] is $14.3 \mathrm{eV}$, and the highest-occupied-to-lowest virtual separation from the $\mathrm{Al}_{4} \mathrm{~N}$ cluster calculation is $8.35 \mathrm{eV}$.

Regarding charge state 1 , although the aluminium ions do not accept any of the four vacancy-centred electrons, either in the ground state or in the excited state, nitrogen orbitals, if they were significantly involved at the bottom of the virtual manifold, might do so. This 
outward shift of electronic charge would then affect the nearest-neighbour equilibrium distance and reduce the vacancy occupancy.

\section{Summary of conclusions}

We have carried out a modelling study of the ground state and optical absorption of the nitrogen vacancy in AlN in charge states 1, 2 and 3. We find the ground state spins to be 1, 1/2 and 0 (units $\hbar$ ). Both ground and unrelaxed excited states principally consist of four, three and two vacancy electrons respectively, with a spin-up-spin-down pair of s-type electrons in each case, and with the aluminium ions maintaining filled-shell configurations in all three charge states. In addition, charge state 1 has two unpaired p-type electrons, and charge state 2 has one such electron. In a system lacking a centre of symmetry, the optical transitions are s-to-p-like for charge state 3, and roughly p to mixed s- and p-type character in charge states 2 and 1 . The expected trend of nearest-neighbour relaxation for the defect as a function of charge is not found. The computational model is discussed critically and at length.

\section{Acknowledgments}

JMV and DKC are grateful for support from the Department of Physics and Astronomy, University of Manitoba, and from the Deep River Science Academy, Pinawa, Manitoba. MAB acknowledges funding from the Spanish MCyT, grant BQU2003-06553. The following assisted with the work: R Orlando, R Collister, A Shields and C Burns. JMV is also grateful for hospitality at Michigan Technological University.

\section{References}

[1] See for example Wetzel C, Gil B, Kuzuhara M and Mantra M (ed) 2005 GaN, AlN, InN and their alloys Materials Research Society Symp. Proc. vol 831 (Warrendale, PA: Materials Research Society)

[2] Stampfl C and Van de Walle C G 2002 Phys. Rev. B 65155212

[3] Harding J H, Harker A H, Keegstra P B, Pandey R, Vail J M and Woodward C 1985 Physica B and C 131151

[4] Dick B G and Overhauser A W 1958 Phys. Rev. 112395

[5] Vail J M, Penner O, Qiu Q C, Xu Y and Yang A, unpublished

[6] Vail J M, Schindel D and Collister R, unpublished

[7] Vail J M, Schindel D, Yang A, Penner O, Pandey R, Jiang H, Blanco M A, Costales A, Qiu Q C and Xu Y 2004 J. Phys.: Condens. Matter 163371

[8] Pandey R, Sutjianto A, Seel M and Jaffe J E 1993 J. Mater. Res. 81922

[9] Bader R F 1990 Atoms in Molecules (Oxford: Oxford University Press)

[10] See for example: Seidel H and Wolf H C 1968 Physics of Color Centers ed W B Fowler (New York: Academic) chapter 8 , section I.B.2

[11] Vail J M, Coish W A, He H and Yang A 2002 Phys. Rev. B 6601409

[12] Vail J M, Chevrier D K, Burns C and Shields A, unpublished

[13] Huzinaga S 1985 Handbook of Gaussian Basis Sets (New York: Academic)

[14] Kuiper P, Kruizinga G, Ghijsen J, Sawatzky G H and Verweij H 1989 Phys. Lett. 62221

Meng J, Jena P and Vail J M 1990 J. Phys.: Condens. Matter 210371

[15] Vail J M, Emberly E, Lu T, Gu M and Pandey R 1998 Phys. Rev. B 57764 